\title{
A Case of Foreign Body Granuloma Caused by Acupoint Catgut Embedding Therapy for Obesity
}

\author{
Furong $\mathrm{Li}^{1,2,3, *}$, Liyuan Zhang ${ }^{4, *}$, Xiaodan Wang ${ }^{1,5}$, Mingyue Wang ${ }^{1,2,3}$ \\ ${ }^{1}$ Department of Dermatology, Peking University First Hospital, ${ }^{2}$ National Clinical Research Center for Skin and Immune Diseases, \\ ${ }^{3}$ Beijing Key Laboratory of Molecular Diagnosis on Dermatoses, ${ }^{4}$ Department of Eight-year Clinical Medical Education, Peking University \\ Third Hospital, ${ }^{5}$ Department of Dermatology, Beijing Youan Hospital, Capital Medical University, Beijing, China
}

\section{Dear Editor:}

Foreign body granuloma (FBG), defined as inflammatory reaction induced by various exogenous or endogenous materials, has quite different manifestations and outcomes in patients with different causes ${ }^{1}$, thus a thorough understanding of the causes is very important for early recognition and treatment of FBG. Acupoint catgut embedding therapy (ACET), a widely used traditional Oriental medicine therapy has rarely been reported as a cause of $\mathrm{FBG}^{2,3}$. Here we report a case of FBG in a female after accepting ACET for obesity.

A 57-year old female presented asymptomatic skin leisons on her abdomen and waist for 8 months. She underwent ACET for obesity 1 month prior to the visit. Physical examination revealed indurated erythema with obscure boundary on her abdomen and waist (Fig. 1). Multiple subcutaneous hard nodules were palpated, ranging from bean-sized to egg-sized with poor mobility and skin was felt warm. Histopathologically, multiple variable-sized histiocytic aggregates were recognized extensively in the whole dermis with a few lymphocytes. Basophilic non-structural intracellular substances were found in the histiocytes. The nuclei of giant cells were diffusely scattered (Fig. 2). White cell count was normal. She was diagnosed as FBG and had tried multiple treatments, including betamethasone intralesional injection and topical corticosteroid without significant improvement. We received the patient's consent form about publishing all photographic materials.

ACET is a traditional Oriental medicine therapy prevailing in East Asian countries, in which suture is embedded into acupoints aseptically ${ }^{2}$. It is believed that the existence of the suture will recruit local immune cells, regulate inflammatory cytokines levels, change the metabolic activity and enhance local circulation ${ }^{3}$. ACET is widely used in several diseases, such as chronic pain, epilepsy, obesity, and constipation et al. ${ }^{2,3}$ In previous researches, suture implantation has been recognized universally as a cause of FBG in surgery ${ }^{4}$. However, little attention was paid to the role of

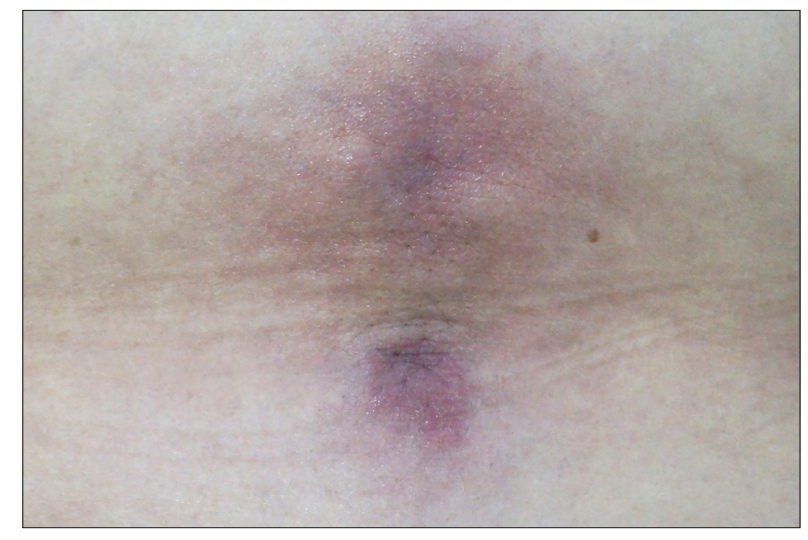

Fig. 1. Clinical presentation. Indurated erythema with obscure boundary is located on her abdomen.

Received July 25, 2019, Revised September 12, 2019, Accepted for publication November 21, 2019

*These authors have equally contributed to the article.

Corresponding author: Mingyue Wang, Department of Dermatology, Peking University First Hospital, 8 Xishiku Street, Xicheng District, Beijing 100034, China. Tel: 86-10-83573075, Fax: 86-10-66551216, E-mail: wangmy@pku.edu.cn ORCID: https://orcid.org/0000-0001-8317-3414

This is an Open Access article distributed under the terms of the Creative Commons Attribution Non-Commercial License (http://creativecommons.org/licenses/by-nc/4.0) which permits unrestricted non-commercial use, distribution, and reproduction in any medium, provided the original work is properly cited.

Copyright (C) The Korean Dermatological Association and The Korean Society for Investigative Dermatology 


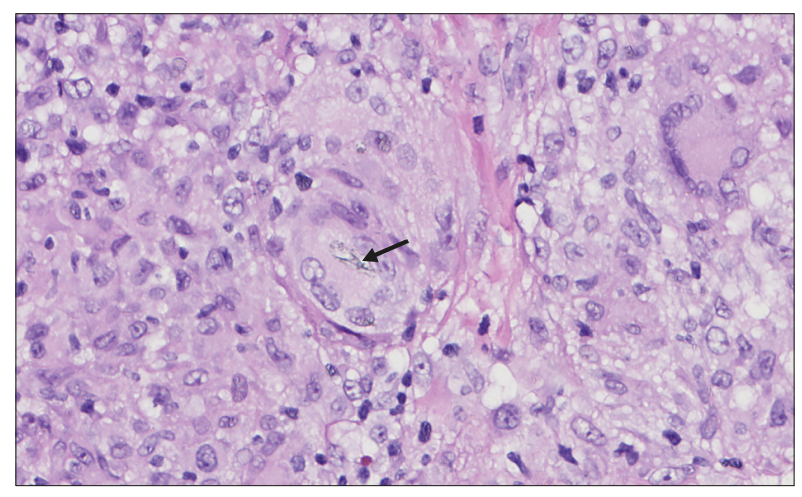

Fig. 2. Histopathology of the lesion. Skin biopsy from the lesion reveals several histiocytic granulomas containing basophilic nonstructural substance (arrow) (H\&E; original magnification, $\times 200$ ).

catguts as a potential cause of FBG in patients who had undergone $\mathrm{ACET}^{2}$. In 2011, Chuang et al. ${ }^{5}$ reported the first case of a patient who developed FBG 1 month after accepting ACET for obesity. In that case, multiple beansized tender itchy erythematous nodules with linear distribution were observed and regressed spontaneously. However, despite several treatments, the skin lesions of our patient did not resolve after 9 months. The rate of the catgut absorption depends on the suture location and status of the wound process determined by the counts and quality of cell population surrounding the catgut ${ }^{4}$. Importantly, the features of FBG induced by ACET are summarized as follows: firstly, a history of accepting ACET; secondly, mainly presenting as erythematous nodules with line arrangement; thirdly, asymptomatic or itchy; lastly, outcome was uncertain because that spontaneous resolution or inefficacious to many therapies could also be observed.

Dermatologists should be aware that ACET may be a cause of FBG when an overweight Asian with multiple indurated erythema and subcutaneous nodules on specific areas of the body comes to seek medical care. Careful history taking is important for the differential diagnoses.

\section{CONFLICTS OF INTEREST}

The authors have nothing to disclose.

\section{FUNDING SOURCE}

None.

\section{DATA SHARING STATEMENT}

Research data are not shared.

\section{ORCID}

Furong Li, https://orcid.org/0000-0001-9563-322X

Liyuan Zhang, https://orcid.org/0000-0002-6117-4384

Xiaodan Wang, https://orcid.org/0000-0002-1104-0345

Mingyue Wang, https://orcid.org/0000-0001-8317-3414

\section{REFERENCES}

1. Molina-Ruiz AM, Requena L. Foreign body granulomas. Dermatol Clin 2015;33:497-523.

2. Sheng J, Jin X, Zhu J, Chen Y, Liu X. The effectiveness of acupoint catgut embedding therapy for abdominal obesity: a systematic review and meta-analysis. Evid Based Complement Alternat Med 2019;2019:9714313.

3. Huo J, Zhao J, Yuan Y, Wang J. [Research status of the effect mechanism on catgut-point embedding therapy]. Zhongguo Zhen Jiu 2017;37:1251-1254. Chinese.

4. Thirumaran $M$, Jackson A. Suture granuloma. Postgrad Med J 2004;80:18.

5. Chuang YT, Li TS, Lin TY, Hsu CJ. An unusual complication related to acupuncture point catgut embedding treatment of obesity. Acupunct Med 2011;29:307-308. 